Review began 01/18/2022 Review ended 01/20/2022 Published 01/24/2022

๑) Copyright 2022

Ansari et al. This is an open access article distributed under the terms of the Creative Commons Attribution License CC-BY 4.0. which permits unrestricted use, distribution, and reproduction in any medium, provided the original author and source are credited.

\section{COVID-19 and Bradyarrhythmias: A Case Report With Literature Review}

\author{
Saad Ali Ansari ${ }^{1}$, Yusra Ansari ${ }^{2,3}$, Tahir Muhammad Abdullah Khan ${ }^{4}$ \\ 1. Internal Medicine, University of California Riverside School of Medicine, Riverside, USA 2. Medicine, University of \\ Kentucky, Bowling Green, USA 3. Internal Medicine, Rawalpindi Medical University, Rawalpindi, PAK 4. Pulmonary and \\ Critical Care Medicine, University of Kentucky, Bowling Green, USA
}

Corresponding author: Saad Ali Ansari, saadars13@gmail.com

\begin{abstract}
We report a case of a 51-year-old male with no past medical history who was admitted for acute hypoxic respiratory failure secondary to COVID-19. During his hospitalization, the patient developed sinus bradycardia and frequent sinus pauses were observed on telemetry. No other cause of his bradyarrhythmia was identified except for his COVID-19 infection. There has been numerous case reports and case series describing different arrhythmias seen in patients infected with COVID-19. We present a case of sinus arrest in a patient with COVID-19 and a review of other case reports describing bradyarrhythmia in COVID-19 patients.
\end{abstract}

Categories: Cardiology, Internal Medicine, Infectious Disease

Keywords: complete heart block, pacemaker, bradyarrhythmia, sinus arrest, sinus pauses, covid-19

\section{Introduction}

The COVID-19 pandemic due to severe acute respiratory syndrome coronavirus 2 (SARS-CoV-2) has affected millions of people worldwide since the emergence of the first case in Wuhan, China, in December 2019. Severe acute respiratory syndrome coronavirus 2 has spike (S) proteins on its surface which are responsible for binding to host cell receptors and fusion of viral and cellular membranes. Angiotensinconverting enzyme 2 (ACE 2) is identified as a functional receptor for the spike protein of SARS-CoV-2. The ACE 2 is expressed in high concentrations in the lung (type II alveolar cells),

heart, esophagus, ilium, kidney, and urinary bladder [1,2]. Patients infected with SARS-CoV-2 manifest symptoms ranging from mild to severe respiratory disease including acute respiratory distress syndrome (ARDS) and can involve multiorgan failure.

Although the respiratory system is the most affected organ system, other organ systems involvement has been frequently reported which may manifest as encephalopathy, a hypercoagulable state with thromboembolic disease, deranged liver enzymes, acute kidney failure, and myocardial injury (myocarditis). Cardiac injury (defined as an increase in high sensitivity troponins T or I level more than the 99th percentile upper reference limit and new ECG or transthoracic echocardiographic changes) has been reported in $49 \%$ of patients with critical illness secondary to COVID-19. The most frequent abnormalities observed were EKG or echocardiographic signs of left ventricle abnormalities (87\%), and right ventricular dysfunction (47\%) which was more common than left ventricular dysfunction (13\%). Other abnormalities observed were pericardial effusion (43\%), new-onset atrial arrhythmias (33\%), left ventricular relaxation impairment (33\%), and left ventricle (LV) systolic dysfunction (13\%) [3].

Among conduction abnormalities, sinus node dysfunction with sinus arrest secondary to COVID-19 is rarely reported in the literature and prognosis and disease course in these patients is unknown yet. We report a case of sinus arrest secondary to COVID-19 infection in a critically ill patient who required a permanent pacemaker.

\section{Case Presentation}

A 51-year-old Caucasian male patient, active cigarette smoker, unvaccinated for COVID-19, and no prior history of any medical illness, presented with worsening shortness of breath. The patient tested positive for COVID-19 a few days prior to presentation. On presentation, patient had a blood pressure of $137 / 80 \mathrm{~mm} \mathrm{Hg}$, heart rate of 93 beats per minute, he was afebrile and was saturating at $92 \%$ on $15 \mathrm{~L}$ of $\mathrm{O} 2$ via a non-rebreather mask. He was alert and awake but was showing signs of respiratory distress using accessory respiratory muscle and was tachypneic. Chest auscultation showed bilateral lower lung crackles with no wheezing. Laboratory tests of the patient are given in Table 1 . 


\section{Cureus}

\begin{tabular}{|c|c|c|}
\hline Laboratory Parameter & Patient's Results & Normal Range \\
\hline White blood count & 24.1 k/uL & 4-11 K/uL \\
\hline Hemoglobin & $15.3 \mathrm{~g} / \mathrm{dl}$ & $13-18 \mathrm{~g} / \mathrm{dl}$ \\
\hline Platelet & 344 K/uL & 140-440 K/uL \\
\hline Serum creatinine & 1.34 mg/dl & $0.66-1.25 \mathrm{mg} / \mathrm{dl}$ \\
\hline Serum bicarbonate & 24 mmol/L & $22-30 \mathrm{mmol} / \mathrm{L}$ \\
\hline Lactic acid & $5.9 \mathrm{mmol} / \mathrm{L}$ & $0.7-2.0 \mathrm{mmol} / \mathrm{l}$ \\
\hline Alanine aminotransferase & $70 \mathrm{U} / \mathrm{L}$ & 0-49 U/L \\
\hline Aspartate aminotransferase & $112 \mathrm{U} / \mathrm{L}$ & $17-36$ U/L \\
\hline Total bilirubin & $1.2 \mathrm{mg} / \mathrm{dl}$ & $0.2-1.0 \mathrm{mg} / \mathrm{dl}$ \\
\hline C-reactive protein (CRP) & 18.9 mg/dl & $0-1.0 \mathrm{mg} / \mathrm{dl}$ \\
\hline Procalcitonin & $0.6 \mathrm{ng} / \mathrm{mL}$ & $<0.15 \mathrm{ng} / \mathrm{mL}$ \\
\hline
\end{tabular}

\section{TABLE 1: Laboratory work-up}

Chest X-ray (CXR) showed bilateral ground-glass infiltrates consistent with COVID -19 pneumonia and left pleural effusion (Figure 1). The EKG showed no acute ischemic changes, PR interval at 184 milliseconds (ms), QTc of $490 \mathrm{~ms}$ ( Figure 2) and troponin were elevated to $0.10 \mathrm{ug} / \mathrm{mL}$ (0-0.034 $\mathrm{ug} / \mathrm{mL}$ ) which subsequently trended down to normal values.

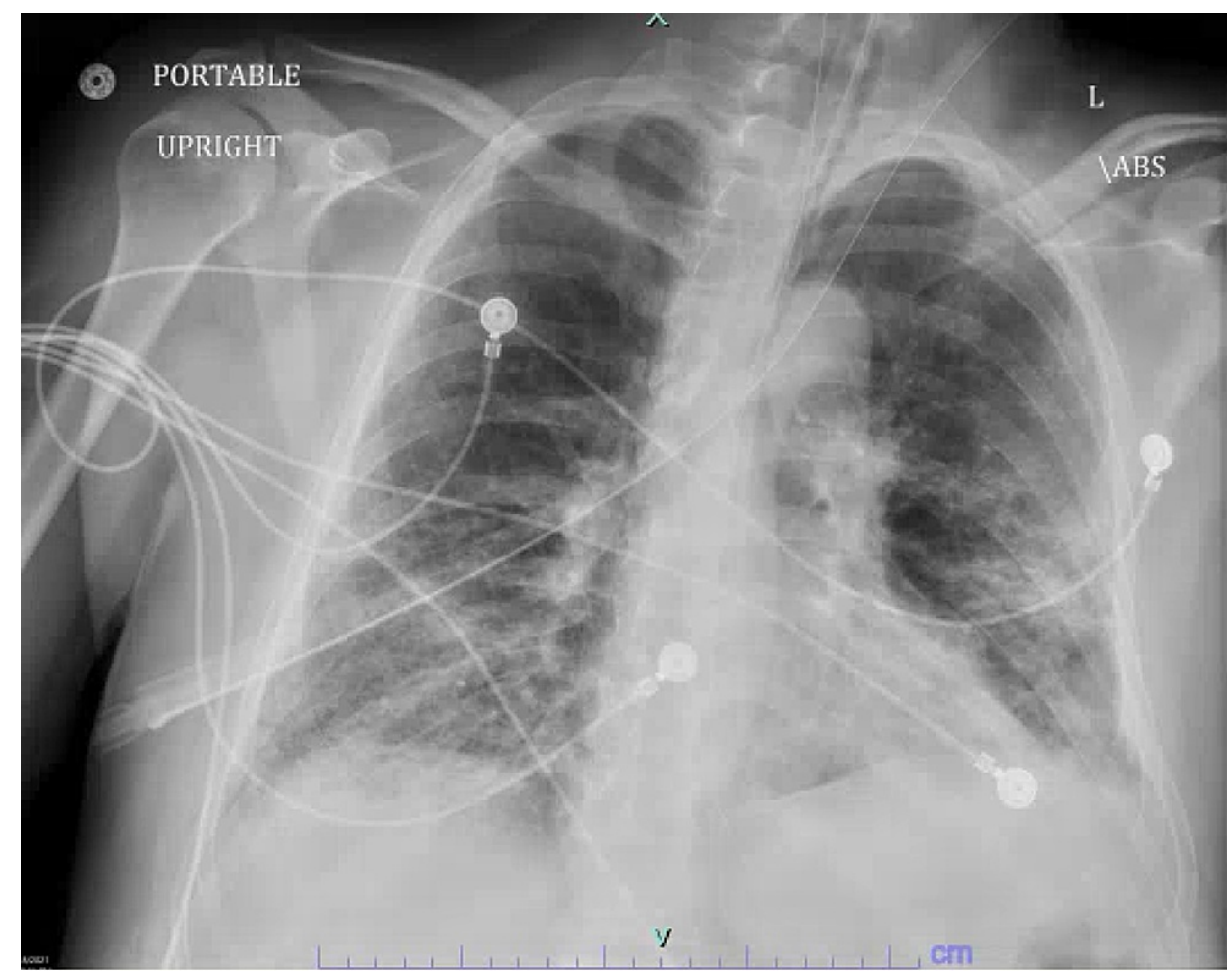

FIGURE 1: Chest X-ray on initial presentation showing bilateral diffuse infiltrates consistent with COVID-19 


\section{Cureus}

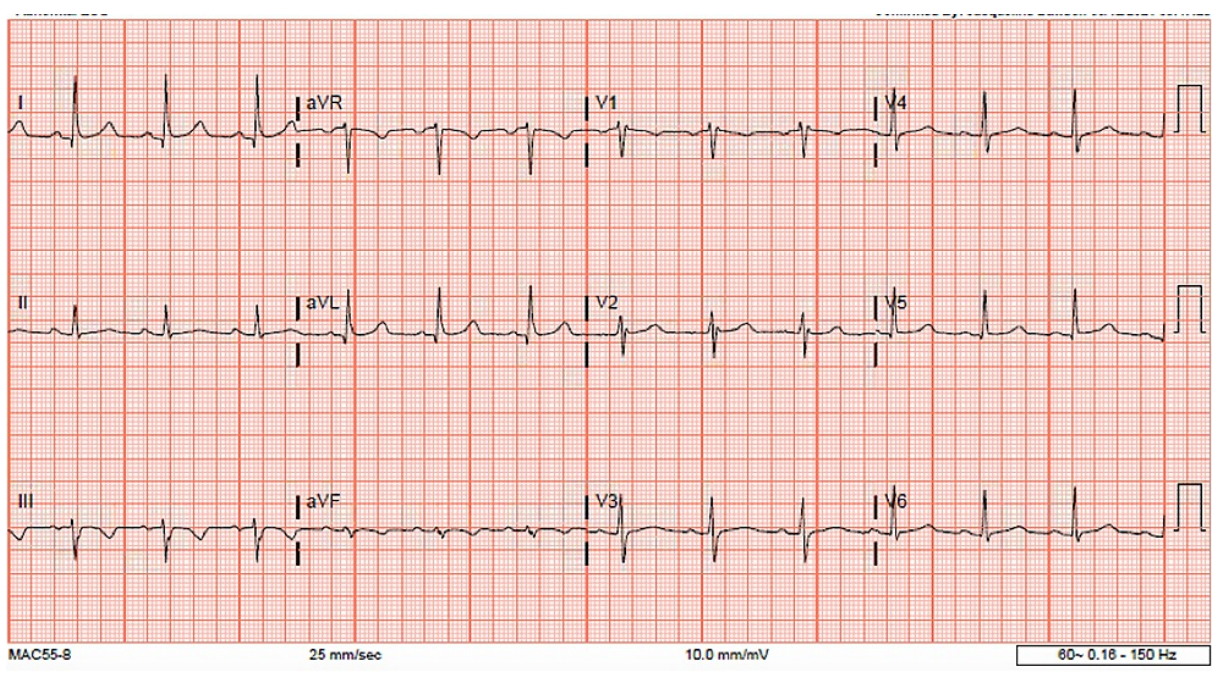

FIGURE 2: EKG on initial presentation

Patient developed respiratory distress in the emergency room and arterial blood gas (ABG) showed PH 7.33, partial pressure of carbon dioxide $(\mathrm{PaCO} 2)$ at 51 , partial pressure of oxygen ( $\mathrm{PaO} 2)$ at 56 , on $100 \%$ fraction of inspired oxygen (FiO2). The patient was intubated and supported with lung protective strategy of mechanical ventilation. Deep tracheal aspirates were sent for gram stain and culture,

and BioFire ${ }^{\circledR}$ FilmArray ${ }^{\circledR}$ (BioFire Inc., Salt Lake City, UT, USA) pneumonia (PN) panel testing post which the patient was initiated on dexamethasone $10 \mathrm{mg}$ twice a day and broad-spectrum antibiotics with linezolid and cefepime. When the sputum culture and pneumonia polymerase chain reaction (PCR) panel did not suggest bacterial infection, the patient was given one dose of $400 \mathrm{mg}$ of sarilumab, and antibiotics were discontinued. Intravenous (iv) propofol and fentanyl infusions were used for sedation and analgesia, and he required a low dose of norepinephrine for sedation induced mild hypotension. Proning protocol was implemented.

Over the following week, the patient developed sinus bradycardia necessitating change of sedatives from propofol to midazolam, and intermittent iv fentanyl for adequate analgesia. Two weeks into his admission, patient developed ventilator associated bacterial pneumonia (VAP) with pan sensitive Escherichia coli which was further complicated by left side pneumothorax warranting placement of 14 French-size pigtail catheters and iv ceftriaxone for VAP. Unfortunately, the patient developed worsening sinus bradycardia with heart rate as low as 27 with frequent sinus pauses observed on telemetry (Figure 3) with largest pause of 12 seconds along with loss of arterial pulse waveform. A transvenous pacemaker was placed.

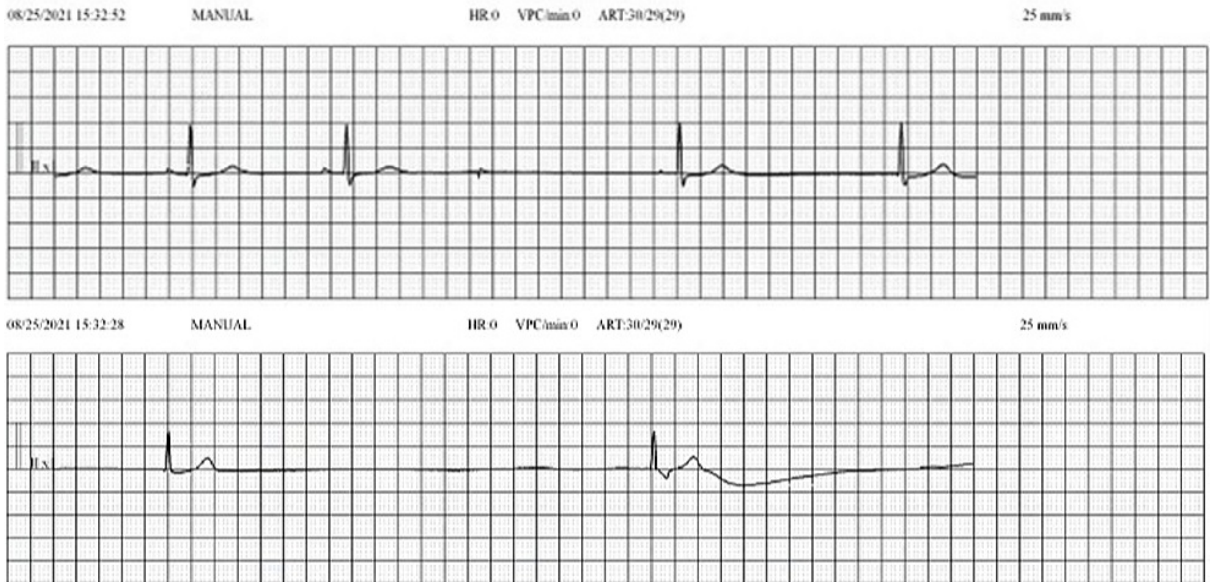

FIGURE 3: Sinus bradycardia with sinus pauses on Telemetry

Telemetry strips showing sinus pauses of approximately 2.2 and $3.6 \mathrm{~s}$.

The EKG showed mild LV hypertrophy with LV ejection fraction of $60 \%$ to $65 \%$ and no regional wall motion abnormality. After treatment of VAP, requirement of supplemental oxygen improved but he could not be 


\section{Cureus}

liberated from mechanical ventilator due to excessive respiratory secretions. Bedside percutaneous tracheostomy was performed without complication, and due to persistent intermittent dependence of transvenous pacemaker, a permanent pacemaker (Figure 4) was placed without complication as per discretion of electrophysiologist. The patient was later transferred to long term acute care facility (LTAC) for weaning from ventilator and physical and occupational therapy. Unfortunately, the patient's condition was complicated by bilateral lower extremities deep venous thrombosis requiring anticoagulation therapy and later by the second event of ventilator associated pneumonia with Stenotrophomonas maltophilia with bacteremia and septic shock. The patient's family opted for comfort care and he succumbed to his illness.

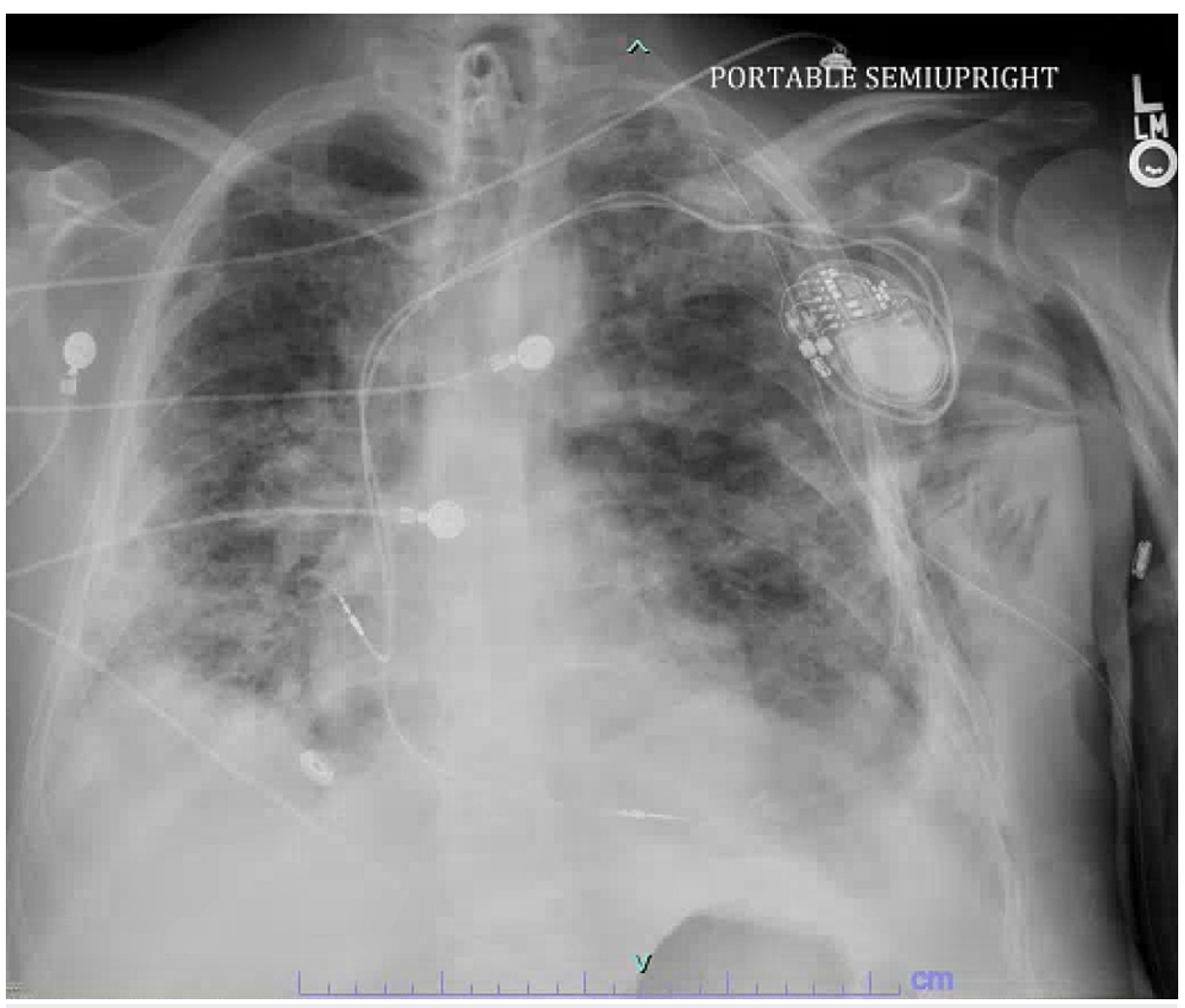

\section{FIGURE 4: Chest X-ray after pacemaker placement}

\section{Discussion}

We reviewed a total of 30 case reports/case series involving a total of 67 patients utilizing advanced search on PubMed. Keywords used for literature review were: "COVID-19", "bradyarrhythmias", "bradycardia", "sinus arrest", "sinus pauses", "atrioventricular (AV) block", "heart block", "complete heart block (CHB)", "sinus node dysfunction". Case reports from the start of the pandemic till January 2022 were reviewed. Out of a total of 67 patients, $54 \%$ were males (36/67) while $46 \%$ were females (31/67). The mean age of patients was $56.3 \pm$ 15.2 , mean age for male patients was $53.29 \pm 15.29$ and mean age of female patients was $60.4 \pm 14.1$. Data regarding comorbidities was available for $65 / 67$ patients, $60 \%$ (39/65) of patients were found to have one or more cardiac risk factors. Baseline EKG information was provided for 41 patients, out of them $71 \%(29 / 41)$ had normal baseline EKG. One important observation was that only a very small percentage of patients who developed bradyarrhythmias eventually required intubation and ventilatory support. Of the 53 patients with available data regarding intubation, only $19 \%(13 / 67)$ were put on ventilatory support with one patient declining intubation. Data regarding troponin level and ECHO reading were available for 93\% (62/67) patients. Only $16 \%(10 / 67)$ had some degree of troponin elevation and only $11 \%(7 / 62)$ had a left ventricular ejection fraction (LVEF) $<50 \%$ on ECHO. Many of the patients experienced more than one type of bradyarrhythmia during their clinical course. The most common bradyarrhythmia among the patients was complete heart block (CHB) in 51\% of patients (34/67) followed by sinus bradycardia in 30\% (20/67), sinus arrest in 17.9\% (12/67), second-degree atrioventricular block (AV) block in 7\% (5/67) and high AV block in 3\% (2/67). Only 30\% (20/67) of patients eventually required a permanent pacemaker while one patient declined permanent pacemaker placement. Around $18 \%$ required temporary pacing. The majority of patients i.e., $52 \%$ (35/67) did not require any type of pacing either permanent or temporary and experienced only transient bradyarrhythmia which resolved on its own. Mortality was reported in only 15\% (10/67) of the patients. Table 2 summarizes the data described above. 


\section{Cureus}

\begin{tabular}{|c|c|c|c|c|c|c|c|c|c|c|}
\hline Reference No. & Age & Gender & Comorbidities & LVEF and Troponin & Intubation & $\begin{array}{l}\text { Day since } \\
\text { admission } \\
\text { when EKG } \\
\text { changes } \\
\text { noted }\end{array}$ & $\begin{array}{l}\text { Baseline EKG } \\
\text { on Admission }\end{array}$ & EKG Changes & Pacemaker & Outcome \\
\hline [4] & 69 & Female & $\begin{array}{l}\text { HTN, DM, CVA, } \\
\text { Asthma, HLD }\end{array}$ & Normal & No & 8 & Normal & 2:1 AV Block, sinus arrest & No & Discharged \\
\hline [4] & 83 & Female & HTN, HLD & $\begin{array}{l}\text { Normal LVEF, Elevated } \\
\text { Trop }\end{array}$ & No & 8 & Normal & Sinus Arrest & No & Discharged \\
\hline $\begin{array}{l}\quad \text { (Case } \\
\text { series of } 7 \\
\text { Patients) }\end{array}$ & $\mathrm{n} / \mathrm{a}$ & $\begin{array}{l}\text { Males } \\
3 / 7 \\
\text { Females } \\
4 / 7\end{array}$ & $\begin{array}{l}\text { HTN 5/7, HLD 1/7, DM } \\
\text { 6/7 Hepatic } \\
\text { Disease/Cirrhosis 2/7 } \\
\text { CVD 1/7, CKD 1/7 }\end{array}$ & $\begin{array}{l}\text { Normal LVEF in all } \\
\text { patients Troponin } \\
\text { elevated in } 2 / 7\end{array}$ & $\mathrm{n} / \mathrm{a}$ & n/a & $\begin{array}{l}\text { First Degree AV } \\
\text { Block } 1 / 7 \text { RBBB } \\
2 / 7, \\
\text { LAHB+RBBB } 1 / 7\end{array}$ & $\begin{array}{l}\text { CHB } 2 / 7 \text {, Sinus } \\
\text { Bradycardia and CHB with } \\
1 / 7,2: 1 \text { AV block } 1 / 7 \text {, Sinus } \\
\text { pauses/sinus arrest } 3 / 7\end{array}$ & $\begin{array}{l}\text { Temporary } \\
\text { Pacemaker 3/7 } \\
\text { Permanent } \\
\text { Pacemaker 4/7 }\end{array}$ & $\begin{array}{l}\text { Patients } \\
\text { died } 5 / 7 \\
\text { Discharged } \\
2 / 7\end{array}$ \\
\hline [6] & 34 & Male & $\begin{array}{l}\text { Bipolar Disorder, } \\
\text { Hypothyroidism }\end{array}$ & Normal & Yes & $\mathrm{n} / \mathrm{a}$ & Normal & Sick sinus syndrome & $\begin{array}{l}\text { Permanent } \\
\text { Pacemaker }\end{array}$ & Discharged \\
\hline [7] & 70 & Female & None & Normal & Yes & 2 & Normal & Sinus Bradycardia & No & Discharged \\
\hline [7] & 81 & Male & $\begin{array}{l}\text { Ascending Aortic } \\
\text { Aneurysm, OSA HTN }\end{array}$ & $\begin{array}{l}\text { Normal LVEF, Elevated } \\
\text { Troponin }\end{array}$ & Yes & 4 & Normal & Sinus Bradycardia & No & Discharged \\
\hline [8] & 67 & Male & HTN, HLD, Ex-Smoker & $\begin{array}{l}\text { Normal LVEF, Elevated } \\
\text { Troponin }\end{array}$ & Yes & 7 & $\begin{array}{l}\text { Sinus } \\
\text { bradycardia with } \\
\text { first degree AV } \\
\text { Block }\end{array}$ & Sinus pauses & $\begin{array}{l}\text { Transcutaneous } \\
\text { pacing only }\end{array}$ & Discharged \\
\hline $\begin{array}{l}\quad[9] \\
\quad \text { (Case } \\
\text { series of } 7 \\
\text { Patients) }\end{array}$ & $n / a$ & $\begin{array}{l}\text { Males } \\
4 / 7 \\
\text { Females } \\
3 / 7\end{array}$ & $\begin{array}{l}\text { DM, HTN, MI 2/7 CHF } \\
1 / 7\end{array}$ & $\begin{array}{l}\text { Normal ECHO 5/7, } \\
\text { LVEF }<502 / 7 \text { Elevated } \\
\text { Troponin } 1 / 7\end{array}$ & $\mathrm{n} / \mathrm{a}$ & 1 & $n / a$ & $\begin{array}{l}\text { CHB 5/7 Sick sinus } \\
\text { syndrome } 2 / 7\end{array}$ & $\begin{array}{l}\text { PPM in } 5 \\
\text { patients with } \\
\text { CHB }\end{array}$ & Discharged \\
\hline [10] & 54 & Male & $n / a$ & Normal LVEF & Yes & 14 & Normal & $\mathrm{CHB}$ & No & $\begin{array}{l}\text { Death while } \\
\text { hospitalized }\end{array}$ \\
\hline [11] & 58 & Female & HTN, DM, CHF & $\begin{array}{l}\text { LVEF } 45-50 \% \text {, Normal } \\
\text { Trop }\end{array}$ & No & 5 & Normal & Sinus bradycardia & $\begin{array}{l}\text { Permanent } \\
\text { Pacemaker }\end{array}$ & Discharged \\
\hline [12] & 72 & Female & HTN, DM & $\begin{array}{l}\text { Normal LVEF, Elevated } \\
\text { Troponin }\end{array}$ & Yes & $n / a$ & n/a & $\begin{array}{l}\text { Sinus bradycardia CHB, } \\
\text { Pulseless arrest }\end{array}$ & $\begin{array}{l}\text { Temporary } \\
\text { Pacemaker }\end{array}$ & Discharged \\
\hline [13] & 47 & Female & None & Normal & No & $n / a$ & $n / a$ & $\begin{array}{l}\text { Sinus bradycardia, Sinus } \\
\text { pauses Junctional escape } \\
\text { rhythm with AV dissociation }\end{array}$ & No & $\begin{array}{l}\text { Not } \\
\text { admitted in } \\
\text { hospital }\end{array}$ \\
\hline [14] & 42 & Female & $\mathrm{DM}, \mathrm{BMI}>30$ & $\begin{array}{l}\text { Normal LVEF, Elevated } \\
\text { Trop }\end{array}$ & No & 2 & $n / a$ & $\mathrm{CHB}$ & No & Discharged \\
\hline [14] & 62 & Male & $\begin{array}{l}\text { HTN, DM, CAD, BMI > } \\
25\end{array}$ & $\begin{array}{l}\text { Normal LVEF, Elevated } \\
\text { Trop }\end{array}$ & No & 1 & n/a & $\mathrm{CHB}$ & $\begin{array}{l}\text { Temporary } \\
\text { Pacemaker }\end{array}$ & Discharged \\
\hline [14] & 61 & Male & HTN, BMI > 25 & Normal & No & 2 & $n / a$ & $\mathrm{CHB}$ & No & Discharged \\
\hline [14] & 64 & Male & HTN, BMI > 25 & Normal & No & 2 & $n / a$ & $\mathrm{CHB}$ & No & Discharged \\
\hline [15] & 75 & Female & None & Normal & Yes & 33 & n/a & $\begin{array}{l}\text { Sinus bradycardia, Sinus } \\
\text { pauses }\end{array}$ & $\begin{array}{l}\text { Permanent } \\
\text { Pacemaker }\end{array}$ & Discharged \\
\hline [16] & 55 & Female & None & Normal & No & 2 & Normal & $\begin{array}{l}\text { Sinus bradycardia, Sinus } \\
\text { pauses }\end{array}$ & No & Discharged \\
\hline [17] & 50 & Female & $\mathrm{BMI}>30$ & Normal & No & n/a & $n / a$ & Sinus bradycardia & $\begin{array}{l}\text { Permanent } \\
\text { Pacemaker }\end{array}$ & Discharged \\
\hline [17] & 65 & Female & HTN, DM & Normal & No & $n / a$ & $\mathrm{n} / \mathrm{a}$ & $\mathrm{CHB}$ & $\begin{array}{l}\text { Permanent } \\
\text { Pacemaker }\end{array}$ & Discharged \\
\hline [17] & 43 & Female & None & Normal & No & $\mathrm{n} / \mathrm{a}$ & $\mathrm{n} / \mathrm{a}$ & CHB & $\begin{array}{l}\text { Temporary } \\
\text { Pacemaker }\end{array}$ & Discharged \\
\hline
\end{tabular}




\section{Cureus}

\begin{tabular}{|c|c|c|c|c|c|c|c|c|c|c|}
\hline [17] & 25 & Male & None & Normal & No & $\mathrm{n} / \mathrm{a}$ & $\mathrm{n} / \mathrm{a}$ & $\mathrm{CHB}$ & No & Discharged \\
\hline [17] & 60 & Male & HTN & Normal & No & $\mathrm{n} / \mathrm{a}$ & $\mathrm{n} / \mathrm{a}$ & $\mathrm{CHB}$ & No & Discharged \\
\hline [17] & 70 & Female & HTN & Normal & No & $\mathrm{n} / \mathrm{a}$ & $\mathrm{n} / \mathrm{a}$ & $\mathrm{CHB}$ & $\begin{array}{l}\text { Permanent } \\
\text { Pacemaker }\end{array}$ & Discharged \\
\hline [18] & 71 & Female & $\begin{array}{l}\text { Parkinson Disease, } \\
\text { Tardive Dyskinesia } \\
\text { DM, Bipolar Disorder }\end{array}$ & Normal & No & $\mathrm{n} / \mathrm{a}$ & $\mathrm{n} / \mathrm{a}$ & $\mathrm{CHB}$ & $\begin{array}{l}\text { Permanent } \\
\text { Pacemaker }\end{array}$ & Discharged \\
\hline [19] & 38 & Female & None & Normal & No & $\mathrm{n} / \mathrm{a}$ & $\mathrm{n} / \mathrm{a}$ & Sinus bradycardia, $\mathrm{CHB}$ & No & Discharged \\
\hline [20] & 48 & Male & None & Normal & No & 1 & $\mathrm{n} / \mathrm{a}$ & CHB & No & Discharged \\
\hline [21] & 36 & Male & None & Normal & No & $\mathrm{n} / \mathrm{a}$ & $\mathrm{n} / \mathrm{a}$ & $\begin{array}{l}\text { Sinus node dysfunction, } \\
\text { sinus pauses/ sinus node } \\
\text { arrest }\end{array}$ & $\begin{array}{l}\text { Permanent } \\
\text { Pacemaker }\end{array}$ & Discharged \\
\hline [22] & 44 & Male & DM & Normal & No & 1 & $\mathrm{n} / \mathrm{a}$ & $\mathrm{CHB}$ & No & Discharged \\
\hline $\begin{array}{l}\text { (Case } \\
\text { series of } 6 \\
\text { Patients) }\end{array}$ & $n / a$ & $\begin{array}{l}\text { Male - } \\
3 / 6 \\
\text { Female } \\
-3 / 6\end{array}$ & HTN - 5/6 & $\begin{array}{l}\text { Normal LVEF - 5/6, EF } \\
-45-50 \%-1 / 6 \\
\text { Normal Trop - } 6 / 6\end{array}$ & No & $6,4,3,4,3,3$ & Normal 6/6 & Sinus Bradycardia 6/6 & No & Discharged \\
\hline [24] & 41 & Male & $\begin{array}{l}\text { Familial Mediterranean } \\
\text { Fever }\end{array}$ & Normal & Yes & 5 & Normal & CHB & No & $\begin{array}{l}\text { Death while } \\
\text { hospitalized }\end{array}$ \\
\hline [24] & 77 & Male & None & Normal & Yes & 7 & Normal & $\mathrm{CHB}$, sinus pause & $\begin{array}{l}\text { Temporary } \\
\text { Pacemaker }\end{array}$ & $\begin{array}{l}\text { Death while } \\
\text { hospitalized }\end{array}$ \\
\hline [24] & 36 & Female & $\mathrm{n} / \mathrm{a}$ & $\begin{array}{l}\text { LVEF } 30 \% \text {, Normal } \\
\text { Troponin }\end{array}$ & Yes & 5 & $\begin{array}{l}\text { Sinus } \\
\text { Tachycardia }\end{array}$ & CHB & No & Discharged \\
\hline [25] & 60 & Male & $\begin{array}{l}\text { HTN, Non-Ischemic } \\
\text { Dilated CMP, COPD } \\
\text { HLD, Cocaine abuse }\end{array}$ & $\begin{array}{l}\text { LVEF-25 \%, Normal } \\
\text { Troponin }\end{array}$ & No & 1 & LBBB & $\begin{array}{l}\text { High Grade AV Block w } \\
\text { RBBB escape morphology }\end{array}$ & No & Discharged \\
\hline [26] & 49 & Male & HTN & Normal & No & $\mathrm{n} / \mathrm{a}$ & $\mathrm{n} / \mathrm{a}$ & $\mathrm{CHB}$ & $\begin{array}{l}\text { Permanent } \\
\text { Pacemaker }\end{array}$ & Discharged \\
\hline [27] & 41 & Male & DM & Normal & No & 2 & Normal & $\begin{array}{l}\text { Paroxysmal AV block, Sinus } \\
\text { arrest }\end{array}$ & $\begin{array}{l}\text { Permanent } \\
\text { Pacemaker }\end{array}$ & Discharged \\
\hline [28] & 53 & Male & None & $\begin{array}{l}\text { Mildly impaired LVEF, } \\
\text { Normal Troponin }\end{array}$ & No & 7 & Normal & $\begin{array}{l}\text { Type } 2 \text { AV block (Mobitz 2), } \\
\text { CHB, High Degree AV } \\
\text { Block }\end{array}$ & $\begin{array}{l}\text { Permanent } \\
\text { Pacemaker }\end{array}$ & Discharged \\
\hline [29] & 23 & Male & $\begin{array}{l}\text { Stage 3b Hodgkin's } \\
\text { Lymphoma }\end{array}$ & $\begin{array}{l}\text { LVEF } 35-40 \% \text {, } \\
\text { Elevated Troponin }\end{array}$ & No & 1 & $\begin{array}{l}\text { Sinus } \\
\text { Tachycardia with } \\
\text { RBBB }\end{array}$ & $\mathrm{CHB}$ & $\begin{array}{l}\text { Temporary } \\
\text { Pacemaker }\end{array}$ & Discharged \\
\hline [30] & 74 & Female & DM & Normal Troponin & $\begin{array}{l}\text { Declined } \\
\text { by patient }\end{array}$ & $\mathrm{n} / \mathrm{a}$ & RBBB w LAFB & $\begin{array}{l}\text { RBBB w LPFB, 2 2n Degree } \\
\text { AV Block (Mobitz Type 2) }\end{array}$ & $\begin{array}{l}\text { Declined by } \\
\text { patient }\end{array}$ & $\begin{array}{l}\text { Death while } \\
\text { hospitalized }\end{array}$ \\
\hline [31] & 82 & Male & HTN, CVA & Normal Troponin & Yes & 1 & Normal & $\mathrm{CHB}$ & No & $\begin{array}{l}\text { Death while } \\
\text { hospitalized }\end{array}$ \\
\hline [31] & 55 & Male & None & $\begin{array}{l}\text { Normal Troponin } \\
\text { initially later becoming } \\
\text { Elevated, RH strain of } \\
\text { bedside ECHO }\end{array}$ & Yes & 6 & $\begin{array}{l}\text { RBBB, ST } \\
\text { depressions at } \\
\text { inferior leads } \\
\text { Present at } \\
\text { baseline }\end{array}$ & 2:1 AV Block & No & Discharged \\
\hline [31] & 43 & Male & None & Normal & Yes & 24 & Normal & $\mathrm{CHB}$ & No & Discharged \\
\hline [32] & 56 & Male & DM & Normal & No & 3 & Normal & CHB & $\begin{array}{l}\text { Temporary } \\
\text { pacemaker }\end{array}$ & Discharged \\
\hline [32] & 48 & Male & HTN & Normal & No & 5 & $\begin{array}{l}\text { T wave } \\
\text { inversions V1- } \\
\text { V2 }\end{array}$ & CHB & No & Discharged \\
\hline
\end{tabular}




\section{Cureus}

\begin{tabular}{|c|c|c|c|c|c|c|c|c|c|c|}
\hline [32] & 57 & Female & None & Normal & No & $\mathrm{n} / \mathrm{a}$ & Normal & $\mathrm{CHB}$ & $\begin{array}{l}\text { Temporary } \\
\text { Pacemaker }\end{array}$ & Discharged \\
\hline [32] & 42 & Female & DM & Normal & No & 3 & $\begin{array}{l}\mathrm{T} \text { wave } \\
\text { inversions lead } \\
\text { 1, aVl and } \mathrm{V} 2\end{array}$ & $\mathrm{CHB}$ & $\begin{array}{l}\text { Temporary } \\
\text { Pacemaker }\end{array}$ & Discharged \\
\hline [33] & 55 & Male & $\begin{array}{l}\text { Hypothyroidism, BMI > } \\
30\end{array}$ & $\mathrm{n} / \mathrm{a}$ & No & 1 & Normal & Sinus Bradycardia & No & Discharged \\
\hline [33] & 60 & Female & None & n/a & No & 1 & Normal & Sinus Bradycardia & No & Discharged \\
\hline [33] & 78 & Female & $\begin{array}{l}\text { Hypothyroidism, CAD, } \\
\text { HTN, HLD, BMI > } 25\end{array}$ & n/a & No & 4 & Normal & Sinus Bradycardia & No & Discharged \\
\hline [33] & 73 & Male & $\begin{array}{l}\text { CAD, HTN, HLD, AS, } \\
\text { BMI >35 }\end{array}$ & n/a & No & 4 & Normal & Sinus Bradycardia & No & Discharged \\
\hline
\end{tabular}

\section{TABLE 2: Table comprising data compiled from published case reports/case series on}

bradyarrhythmia's seen in patients with COVID-19 infection.

n/a: Data not available, HTN: Hypertension, DM: Diabetes mellitus, HLD: Hyperlipidemia, CVA: Cerebrovascular accident, LVEF: Left ventricular ejection fraction, CHB: Complete heart block, PPM: Permanent pacemaker, AV: Atrioventricular, CAD: Coronary artery disease, AS: Aortic stenosis, BMI: Body mass index, CMP: Cardiomyopathy, COPD: Chronic obstructive pulmonary disease, CHF: Congestive heart failure, MI: Myocardial infarction, OSA: Obstructive sleep apnea, CVD: Cardiovascular diseases, aVL: Augmented vector left, RBBB: Right bundle branch block, LAFB: Left anterior fascicular block, LPFB: Left posterior fascicular block, LAHB: Left anterior hemiblock

We presented a case of a patient without any major comorbidities who was admitted to the hospital for COVID-19 pneumonia and later during hospitalisation developed sinus bradycardia with frequent sinus pauses for which a permanent pacemaker had to be placed. In our patient who had no significant cardiac risk factors, sinus bradycardia and pauses are possibly related to conduction abnormalities caused by COVID- 19 . COVID-19 has been associated with myocarditis, myocardial infarction, hypercoagulable state, arrhythmias, and conduction abnormalities [34]. From our literature review, it is evident that a lot of patients with COVID-19 who develop bradyarrhythmia have little or no cardiac risk factors. The extent of respiratory compromise or critical illness does not always correlate with conduction abnormalities as only $19 \%$ of patients who developed bradyarrhythmia in our review were intubated and put on ventilatory support. Long term effects of COVID-19 on the conduction system is still to be studied. In the majority of patients, only transient bradycardia or conduction abnormalities were noted. However, up to $30 \%$ of patients did end up getting permanent pacemaker placement. Outpatient follow-ups of patients who develop arrhythmias while hospitalized for COVID-19 infection is necessary to study the long term effects on the cardiac conduction system.

\section{Conclusions}

In conclusion, COVID-19 is associated with wide-ranging cardiac manifestations including bradyarrhythmias. These bradyarrhythmias do not always correlate with severe COVID-19 infection and can present in individuals without any cardiac risk factors. In the majority of patients, these are only transient and resolve as COVID-19 infection subsides, but further study is required to determine the long-term effects of COVID-19 on the conduction system of the heart.

\section{Additional Information}

\section{Disclosures}

Human subjects: Consent was obtained or waived by all participants in this study. Conflicts of interest: In compliance with the ICMJE uniform disclosure form, all authors declare the following: Payment/services info: All authors have declared that no financial support was received from any organization for the submitted work. Financial relationships: All authors have declared that they have no financial relationships at present or within the previous three years with any organizations that might have an interest in the submitted work. Other relationships: All authors have declared that there are no other relationships or activities that could appear to have influenced the submitted work.

\section{References}

1. Li W, Moore MJ, Vasilieva N, et al.: Angiotensin-converting enzyme 2 is a functional receptor for the SARS coronavirus. Nature. 2003, 426:450-454. 10.1038/nature02145

2. Zou X, Chen K, Zou J, Han P, Hao J, Han Z: Single-cell RNA-seq data analysis on the receptor ACE2 
expression reveals the potential risk of different human organs vulnerable to 2019-nCoV infection. Front Med. 2020, 14:185-192. 10.1007/s11684-020-0754-0

3. Doyen D, Dupland P, Morand L, et al.: Characteristics of cardiac injury in critically ill patients with coronavirus disease 2019. Chest. 2021, 159:1974-1985. 10.1016/j.chest.2020.10.056

4. Babapoor-Farrokhran S, Batnyam U, Wiener PC, Kanjanahattakij N, Khraisha O, Amanullah A, Mainigi SK: Atrioventricular and sinus node dysfunction in stable COVID-19 patients . SN Compr Clin Med. 2020, 1-4. 10.1007/s42399-020-00497-5

5. Chinitz JS, Goyal R, Harding M, et al.: Bradyarrhythmias in patients with COVID-19: marker of poor prognosis?. Pacing Clin Electrophysiol. 2020, 43:1199-1204. 10.1111/pace.14042

6. Cimino G, Pascariello G, Bernardi N, et al.: Sinus node dysfunction in a young patient with COVID-19. JACC Case Rep. 2020, 2:1240-1244. 10.1016/j.jaccas.2020.05.067

7. Peigh G, Leya MV, Baman JR, Cantey EP, Knight BP, Flaherty JD: Novel coronavirus 19 (COVID-19) associated sinus node dysfunction: a case series. Eur Heart J Case Rep. 2020, 4:1-6. 10.1093/ehjcr/ytaa132

8. Wang LY, Ng GY: COVID-19 treatment with lopinavir-ritonavir resulting in sick sinus syndrome: a case report. Eur Heart J Case Rep. 2020, 4:1-6. 10.1093/ehjcr/ytaa171

9. Gupta MD, Qamar A, Mp G, et al.: Bradyarrhythmias in patients with COVID-19: a case series . Indian Pacing Electrophysiol J. 2020, 20:211-212. 10.1016/j.ipej.2020.08.004

10. Azarkish M, Laleh Far V, Eslami M, Mollazadeh R: Transient complete heart block in a patient with critical COVID-19. Eur Heart J. 2020, 41:2131. 10.1093/eurheartj/ehaa307

11. Ali M, Awadelkarim A, Bishop P, et al.: Severe sinus bradycardia: an unusual cardiac manifestation of COVID-19. J Investig Med High Impact Case Rep. 2021, 9: 10.1177/23247096211013185

12. Mohamed A: Complete heart block secondary to COVID-19. Cureus. 2021, 13:e14970. 10.7759/cureus.14970

13. Gatto MC, Persi A, Tung M, Masi R, Canitano S, Kol A: Bradyarrhythmias in patients with SARS-CoV-2 infection: a narrative review and a clinical report. Pacing Clin Electrophysiol. 2021, 44:1607-1615. 10.1111/pace. 14308

14. Dagher L, Wanna B, Mikdadi G, Young M, Sohns C, Marrouche NF: High-degree atrioventricular block in COVID-19 hospitalized patients. Europace. 2021, 23:451-455. 10.1093/europace/euaa333

15. Elices-Teja J, Bueno-Sacristán Á, Durán-Bobin O, Ferreiro-González AM, Gonzalez-Juanatey C: Sinus node syndrome in a critical COVID-19 patient. Rev Port Cardiol (Engl Ed). 2021, 10.1016/j.repc.2020.09.015

16. Olagunju A, Forst B, Yakymovych O, Yeneneh BT: Multiple sinus pauses in a patient with COVID-19 . Cureus. 2021, 13:e14040. 10.7759/cureus.14040

17. Amir M, Yoseph H, Farisi AT, Phie JK, Adam AT: Symptomatic bradycardia in Covid-19 hospitalized patients: a case series. Int J Infect Dis. 2021, 111:1-4. 10.1016/j.ijid.2021.07.068

18. Haddadin FI, Mahdawi TE, Hattar L, Beydoun H, Fram F, Homoud M: A case of complete heart block in a COVID-19 infected patient. J Cardiol Cases. 2021, 23:27-30. 10.1016/j.jccase.2020.08.006

19. Dehghani Firouzabadi M, Goudarzi S, Dehghani Firouzabadi F, Moosaie F: Complete heart block and itchy rash in a patient with COVID-19. Caspian J Intern Med. 2020, 11:569-571. 10.22088/cjim.11.0.569

20. Hosseini Z, Ghodsi S, Hejazi SF: Persistent complete heart block in a patient with COVID-19 infection: a case report. SN Compr Clin Med. 2021, 1-4. 10.1007/s42399-020-00712-3

21. Eid MM: COVID-19 patient with symptomatic bradycardia. Vis J Emerg Med. 2021, 22:100920. 10.1016/j.visj.2020.100920

22. Chen JH, Robinson B, Patel P, Kata P, Kanukuntla AK, Okere A, Cheriyath P: Transient complete heart block in a patient with COVID-19. Cureus. 2021, 13:e15796. 10.7759/cureus.15796

23. Srinivasan A, Pansuriya T, Wilson B, et al.: Systemic inflammation-related bradycardia in COVID-19. Case Rep Cardiol. 2021, 2021:9986955. 10.1155/2021/9986955

24. Ahmad F, Gandre P, Nguekam J, et al.: Complete heart block as a clinical feature in critically ill coronavirus disease 2019 (COVID-19) patients: a case series of three cases. Case Rep Crit Care. 2021, 2021:9955466. $10.1155 / 2021 / 9955466$

25. Jean-Louis F, Adedayo AM, Ajibawo T, Upadhya G, Syed A: A rare case of resolution of high-degree atrioventricular block associated with COVID-19. J Med Cases. 2020, 11:243-245. 10.14740/jmc3524

26. Gyawali B, Baral B, Shah S, Yadav S, Poudel CM: A patient infected with SARS-CoV-2 presenting with complete heart block. Case Rep Cardiol. 2021, 2021:5011294. 10.1155/2021/5011294

27. Abe M, Chiba S, Kataoka S, et al.: Paroxysmal atrioventricular block in a relatively young patient with COVID-19. Intern Med. 2021, 60:2623-2626. 10.2169/internalmedicine.6237-20

28. Ashok V, Loke WI: Case report: high-grade atrioventricular block in suspected COVID-19 myocarditis . Eur Heart J Case Rep. 2020, 4:1-6. 10.1093/ehjcr/ytaa248

29. Bhasin V, Carrillo M, Ghosh B, Moin D, Maglione TJ, Kassotis J: Reversible complete heart block in a patient with coronavirus disease 2019. Pacing Clin Electrophysiol. 2021, 44:1939-1943. 10.1111/pace.14321

30. Gubitosa JC, Xu P, Ahmed A, Pergament K: Incomplete trifascicular block and Mobitz type II atrioventricular block in COVID-19. Cureus. 2020, $12:$ e10461. 10.7759/cureus.10461

31. Eneizat Mahdawi T, Wang H, Haddadin FI, Al-Qaysi D, Wylie JV: Heart block in patients with coronavirus disease 2019: a case series of 3 patients infected with SARS-CoV-2. HeartRhythm Case Rep. 2020, 6:652656. 10.1016/j.hrcr.2020.06.014

32. Sharif MH, Khan AW, Khaleeque M, et al.: Complete heart block in patients infected with SARS-CoV-2: a case series from a developing country. Ann Med Surg (Lond). 2021, 69:102828. 10.1016/j.amsu.2021.102828

33. Amaratunga EA, Corwin DS, Moran L, Snyder R: Bradycardia in patients with COVID-19: a calm before the storm?. Cureus. 2020, 12:e8599. 10.7759/cureus.8599

34. Long B, Brady WJ, Koyfman A, Gottlieb M: Cardiovascular complications in COVID-19. Am J Emerg Med. 2020, 38:1504-1507. 10.1016/j.ajem.2020.04.048 